\title{
A physical model for formation and evolution of QSOs and of their spheroidal hosts
}

\author{
Luigi Danese*, Michele Cirasuolo, Francesco Shankar \\ SISSA, Italy \\ E-mail: danesedsissa.it
}

Gian Luigi Granato, Gianfranco De Zotti

INAF - Osservatorio Astronomico di Padova, Italy

\section{Laura Silva}

INAF - Osservatorio Astronomico di Trieste, Italy

\begin{abstract}
We present a physical model for the coevolution of massive spheroidal galaxies and active nuclei at their centers. Supernova heating is increasingly effective in slowing down the star formation and in driving gas outflows in smaller and smaller dark matter halos. Thus the more massive protogalaxies virializing at early times are the sites of faster star formation. In the central galactic regions the correspondingly higher radiation drag due to starlight acts on the cold gas, further decreasing its angular momentum and causing an inflow towards the central $\mathrm{BH}$, increasing its mass and powering the nuclear activity. In turn, the feedbacks from SN explosions and in particular from the active nucleus regulate the star formation rate and the gas inflow, and eventually unbind the residual gas, thus halting both the star formation and the $\mathrm{BH}$ growth, in a time shorter for larger halos. The model accounts for a broad variety of dynamical, photometric and metallicity properties of early-type galaxies, for the local supermassive black-hole mass function and for the relation between the $M_{\mathrm{BH}}$ and $\sigma$, as well as an even tighter correspondence between $M_{\mathrm{BH}}$ and the mass in stars.
\end{abstract}

Baryons in Dark Matter Halos

5-9 October 2004

Novigrad, Croatia

${ }^{*}$ Speaker. 


\section{Introduction}

There is growing evidence that massive galaxies at high redshifts are far more numerous than predicted by standard semi-analytic models (Scott et al. 2002; Daddi et al. 2003; Tecza et al. 2004). Also, the $[\alpha / F e]$-magnitude relation points towards a higher abundance of $\alpha$-elements for more luminous/massive galaxies, indicating that the time for type Ia SNe to enrich the ISM must have been shorter for the more massive systems. Direct evidence that massive galaxies with $M \gtrsim 10^{11} M_{\odot}$ were in place at $z \gtrsim 2$, is also provided by recent $K$-band surveys (Kashikawa et al. 2003; Fontana et al. 2004). Moreover, the space density of Extremely Red Objects (EROs) at $z \gtrsim 3$ is only a factor $\sim 5-10$ less than at $z \sim 1$ (Tecza et al. 2004). Finally, extremely massive black holes $(\mathrm{BH})$, with $\log \left(M_{\mathrm{BH}} / M_{\odot}\right)>8-9$, must have formed very quickly at early cosmic times to power highly luminous quasars at redshift of up to $>6$ (Fan et al. 2001).

It is also clear that the nuclear activity - powered by accretion onto super-massive $\mathrm{BH}-$ is also intimately related to the formation of the host galaxy. The presence of massive BHs in almost all local galaxies with a spheroidal component and the tight correlations between the mass of these central objects and dynamical and photometric properties of galactic bulges support this connection (Ferrarese \& Merritt 2000; Gebhardt et al. 2000; McLure \& Dunlop 2002).

The idea that supernova (SN) and QSO feedback play an important role in the evolution of spheroidal galaxies has been pointed out by several authors (Dekel \& Silk 1986; Haehnelt, Natarajan \& Rees 1998; Silk \& Rees 1998; Fabian 1999). Granato et al. (2004) have shown that the feedback from SN explosions and from nuclear activity can reverse the hierarchical scenario for baryons. In other words, the canonical hierarchical Cold Dark Matter (CDM) scheme - small clumps collapse first - is reversed for baryon collapse and the formation of luminous objects (Antihierarchical Baryon Collapse (ABC) scenario). In the following we briefly describe this model and summarize some of its predictions.

\section{The Model}

The ABC model applies to massive spheroidal galaxies and galactic bulges with halo mass $\log \left(M_{\mathrm{vir}} / M_{\odot}\right)>11.4$, virializing at $z \geq 1.5$. At this high redshift a good approximation of the virialization rate of these objects is given by the positive term of the derivative of the halo mass function as given by Press \& Schechter 1974 and revised by Sheth \& Tormen 1999. Numerical simulations (see e.g. Zhao et al. 2003) have shown that the build-up of DM halos consists of an early phase of fast accretion, during which there is a rapid increase of the specific binding energy and of the central potential well, and of a late phase of slow accretion with no significant change of the binding energy and of the circular velocity. The fast accretion phase is dominated by major mergers, which favor the loss of angular momentum by dynamical friction $\left(t_{D F} \leq t_{d y n}\right)$.

The diffuse gas within the DM halo, shock heated to the virial temperature of the halo and moderately clumpy (clumping factor $\simeq 20$ ), cools to form stars, especially in the high density regions, at a rate ruled by the cooling and dynamic timescales. In small halos a few $\mathrm{SNe}$ are sufficient to quench the star formation, while in the big ones nothing prevents a huge starburst ( $\geq 1000 M_{\odot} /$ yr over $0.5-0.8 \mathrm{Gyr}$ ), which occurs in clouds heavily obscured by dust and yields extremely large submillimeter luminosities (SCUBA phase). 


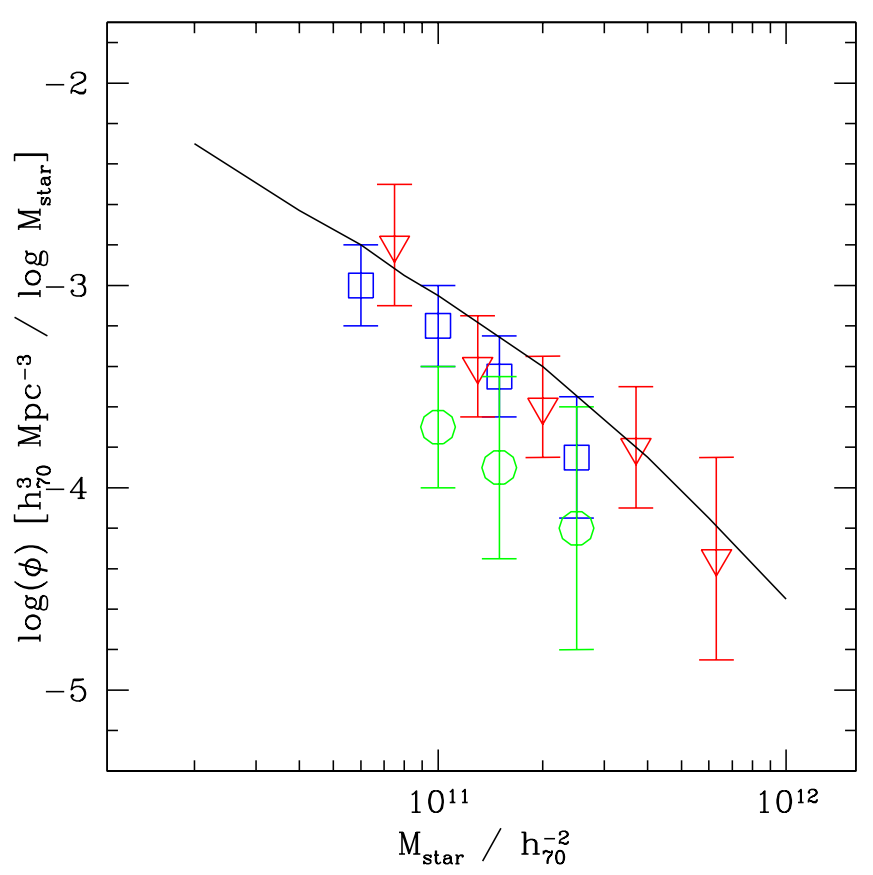

Figure 1: Stellar mass functions of galaxies in the range $1.5 \leq z<2$ derived by Fontana et al (2004) (different symbols correspond to different methods to estimate the stellar mass), compared with theoretical prediction of the $\mathrm{ABC}$ model (solid line).

In the central galactic regions the radiation drag due to starlight acts on the cold gas (Kawakatu \& Umemura 2002), further decreasing its angular momentum and causing an infbw into a reservoir around the central super-massive $\mathrm{BH}(\mathrm{SMBH})$, to be subsequently accreted into it, increasing its mass and powering the nuclear activity. The growth proceeds until nuclear feedback is strong enough to unbind the residual ISM, thus halting both the star formation and the BH growth, letting the active nucleus shine unobscured (QSO phase). The time required to sweep out the ISM decreases with increasing halo mass, thus accounting for the $[\alpha / F e]$-magnitude relation. An almost passive evolution of the stellar population, with a dormant $\mathrm{SMBH}$, follows (EROs phase).

\section{Results}

As shown by Granato et al. (2004), the ABC model, coupled with the spectro-photometric code GRASIL by Silva et al. (1998), accounts for a broad variety of data, including the SCUBA counts at $850 \mu \mathrm{m}$, the corresponding preliminary redshift distribution and the local K-band luminosity function of massive spheroidal galaxies. In Fig. 1 we compare the model predictions with the distribution of stellar masses in galaxies up to $z \simeq 2$ determined by Fontana et al. (2004). At $z \sim 3$ the model predicts a comoving number density of galaxies with masses $M_{\text {star }} \geq 10^{11} M_{\odot}$ of $n \sim 10^{-4} / \mathrm{Mpc}^{3}$ in agreement with the lower limit found by Tecza et al. (2004) (Fig. 2). In fact the ABC model predicts SFRs and masses in stars in massive virialized DM halos significantly larger than other semi-analytical models, whose predictions fall well below the observational upper limit. 


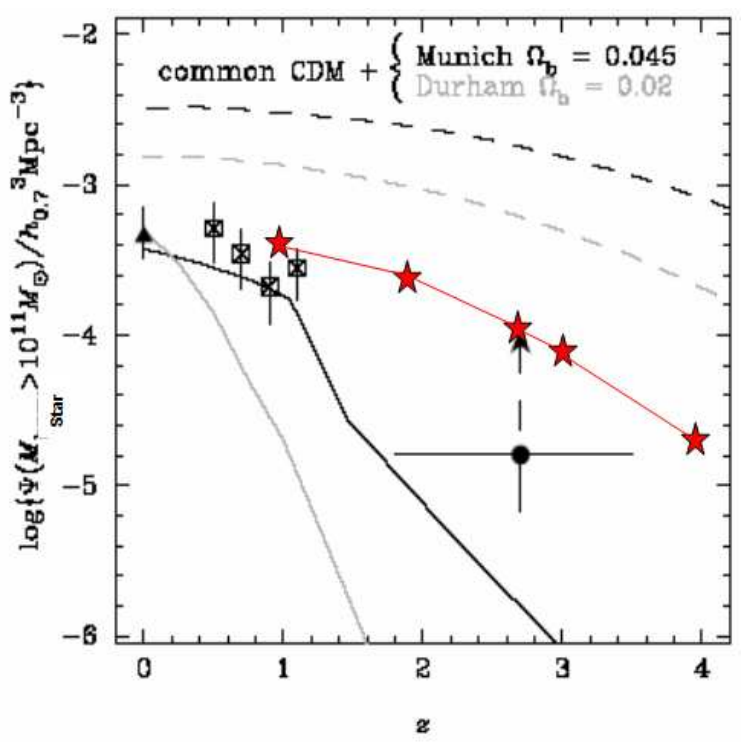

Figure 2: Comoving number densities of galaxies with baryonic masses $\geq 10^{11} M_{\odot}$ as a function of redshift. The triangle and open rectangles show densities of massive stellar systems at $z=0$ (Cole et al. 2001) and $z \sim 1$ (Drory et al. 2004). The circle with upward arrow is the lower limit by Tecza et al. (2004). The solid curves show the predictions of Kauffmann et al. (1998) (upper) and Baugh et al. (2003); the dashed curves show the number densities of halos with available baryonic masses $\geq 10^{11} M_{\odot}$ for the values of $\Omega_{b}$ adopted by Kauffmann et al. (1998) (upper) and Baugh et al. (2003). The line with stars is the ABC model prediction. Adapted from Tecza et al. (2004)

The star formation history of spheroidal galaxies is also mirrored in the dynamical and photometric properties of these systems, as can be determined by the velocity dispersion function (VDF) and the Fundamental Plane (FP). A crucial problem is how to connect the 'observed' velocity dispersion $(\sigma)$ to the 'theoretical' virial velocity $\left(V_{\text {vir }}\right)$, which, for a given cosmology, characterizes dynamical properties of a halo of mass $M_{\mathrm{vir}}$ virializing at $z_{\mathrm{vir}}$. The velocity functions of halos virializing at any given redshift, predicted by the standard hierarchical clustering model for galaxy formation, can be straightforwardly computed from their mass function. Integrating over redshift, for $z_{\mathrm{vir}} \geq 1.5$, the local velocity function of spheroids can be obtained.

Adopting a constant ratio $\sigma / V_{\text {vir }}=0.55$, consistent with the observational results (Gerhard et al. 2001; Ferrarese 2002), Cirasuolo et al. (2004) have found a very good match to the velocity dispersion function (Fig. 3) of early-type galaxies and bulges derived by Sheth et al. (2003), using SDSS data (Bernardi et al. 2003). This result strongly suggests that dissipative processes during the settling of the baryonic component and later merging events had little impact on the stellar dynamics.

The $V_{\text {vir }}-\sigma$ relation is a key ingredient to connect theoretical predictions with observations. Using the above determination of the $\sigma / V_{\text {vir }}$ ratio, we have shown that the observed relationships between photometric and dynamical properties of spheroidal galaxies, defining the Fundamental Plane, carry clear imprints of the feedback processes ruling the early evolution of spheroidal galaxies (Cirasuolo et al. 2004). As shown in Figure 4, the treatment of feedback adopted in the ABC 


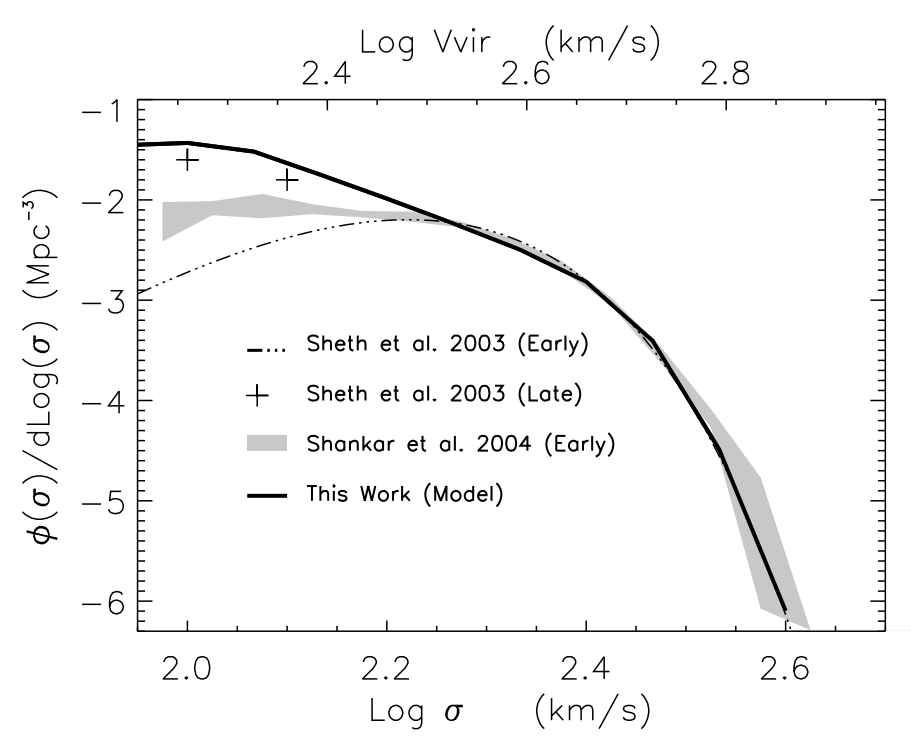

Figure 3: Estimates of the velocity dispersion function of early-type galaxies by Shankar et al. (2004) (shaded area) and Sheth et al. (2003) (triple-dot dashed line). The crosses show the contribution of bulges of late-type galaxies, determined by Sheth et al. (2003). The solid line shows the model virial velocity function (upper scale). The two functions match for $\sigma=0.55 V_{\text {vir }}$.

model nicely accounts not only for the slope, but also for the normalization of the luminosity- $\sigma$ (Faber-Jackson) relation for SDSS galaxies (Bernardi et al. 2003). The model implies that the observed scatter is mostly intrinsic and due to the spread of virialization redshifts. The effect of feedbacks also accounts for the observed luminosity-effective radius relation.

Cirasuolo et al. (2004) have also shown that the parameters controlling the transfer of the energy from SNae and from AGN winds to the gas in the DM halo are well constrained by available observations of the local luminosity function, FP and $M_{\mathrm{BH}}-\sigma$ relationship of spheroidal galaxies. A major consequence of such feedbacks is that only a small fraction of baryons initially available inside the DM halos are eventually found in stars, in agreement with the common consent that $\Omega_{\text {star }} \leq 0.1 \Omega_{\text {bar }}$.

The evolution of the stellar component is tied to that of the central black hole, and therefore the $M_{\mathrm{BH}}-\sigma$ relation is also shaped by the effect of feedback, primarily from the active nucleus itself in the more massive systems, and from supernovae in smaller objects. Again, observations are well reproduced (see left panel of Fig. 5), the dispersion around the best-fit relation is expected to be largely due to the different virialization redshifts. A steepening of the $M_{\mathrm{BH}}-\sigma$ is also expected for low BH masses, due to the combined effect of SN feedback - which is increasingly efficient with decreasing halo mass in slowing down the gas infall onto the central $\mathrm{BH}-$ and of the decreased radiation drag.

The model predicts that the relation between $M_{\mathrm{BH}}$ and the mass in stars is essentially redshift independent, since these two masses grow in parallel. Therefore, the $M_{\mathrm{BH}}-M_{\text {star }}$ relation is expected to have a smaller intrinsic scatter than the $M_{\mathrm{BH}}-\sigma$ relation (see right panel of Fig. 5), consistent with recent observations (Marconi \& Hunt 2003; Häring \& Rix 2004). The local supermassive BH mass function and its antihierachical assembling found by Shankar et al. (2004) are well reproduced by the model. 


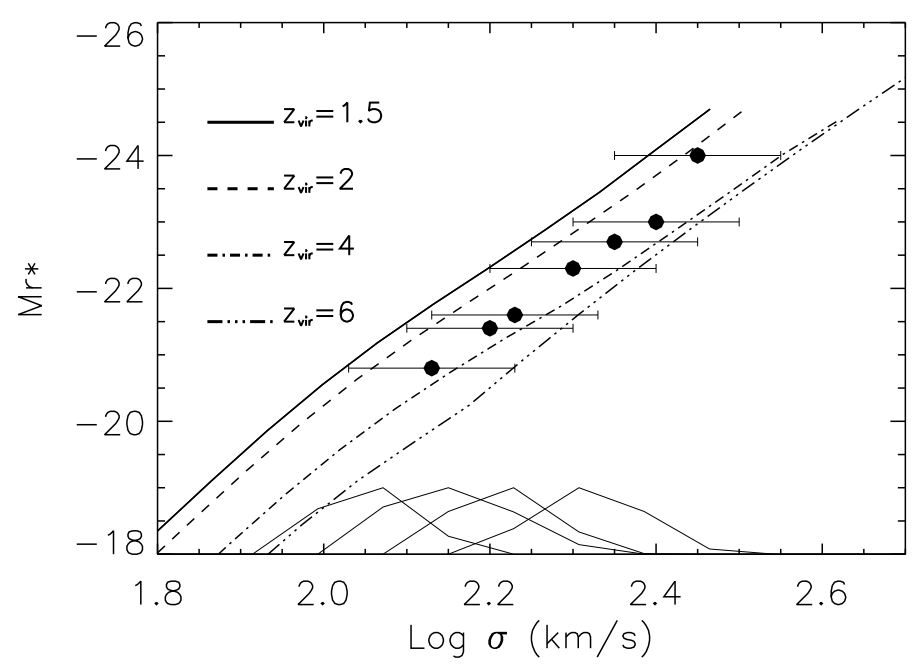

Figure 4: Observed Faber-Jackson relation (fi lled dots) from Bernardi et al. (2003), compared with our predictions for different virialization redshifts $z_{\mathrm{vir}}$. The curves at the bottom of the fi gure represent the normalized distributions of velocity dispersions of galaxies in 4 absolute magnitude bins 0.5 mag wide, centered at $M r *=-20.2,-21,-22$, and -23 (from left to right), as predicted by the ABC model. The FWHMs of the predicted distributions are remarkably close to the observed values (FWHM $\sim 0.09$; Bernardi et al. 2003)

\section{Summary and conclusions}

In our model the Anti-hierarchical growth of the baryonic component in DM halos is a natural result of the feedbacks. The heating from SNe is increasingly effective in slowing down the star formation and driving gas outfbws in shallower potential wells. As a consequence, the star formation is faster within the most massive halos. A higher star formation implies a higher radiation drag and therefore a faster SMBH fueling and growth. The model also implies a stronger AGN kinetic output for massive systems $\left(\propto M_{B H}^{3 / 2}\right)$ causing an earlier sweeping out of the ISM. Thus the duration of the starburst and of the SMBH growth is shorter for more massive halos $(\leq 1 \mathrm{Gyr}$ for $M_{\text {halo }} \geq 10^{12} M_{\odot}$ and $3 \leq z_{\text {vir }} \leq 6$ ).

During the intense starburst and SMBH growth phase the spheroid is heavily dust obscured (SCUBA phase); the model indeed fits the SCUBA counts and the (albeit limited) data on the redshift distribution. We expect the hard X-ray emission recently detected from SCUBA galaxies by Alexander et al. (2003) $\left(L_{X} \sim 10^{43}-10^{44} \mathrm{erg} / \mathrm{s}\right)$ is associated to the growing phase of the central $\mathrm{SMBH}$. Dust prevents us to detect this phase at optical wavelengths.

When the AGN reaches its maximum power, the ISM is blown away and the AGN shines unobscured (QSO phase). The gas surrounding powerful high- $z$ QSOs is therefore expected to exhibit at least solar abundances and $\alpha$-enhancement, as indeed found by D'Odorico et al. (2004).

Afterwards the spheroids evolve passively (ERO phase) (see for a comprehensive discussion Silva et al 2004). The model reproduces the mass and redshift distributions of sources detected by deep K-band surveys, which proved to be extremely challenging for all the other semi-analytical models. 

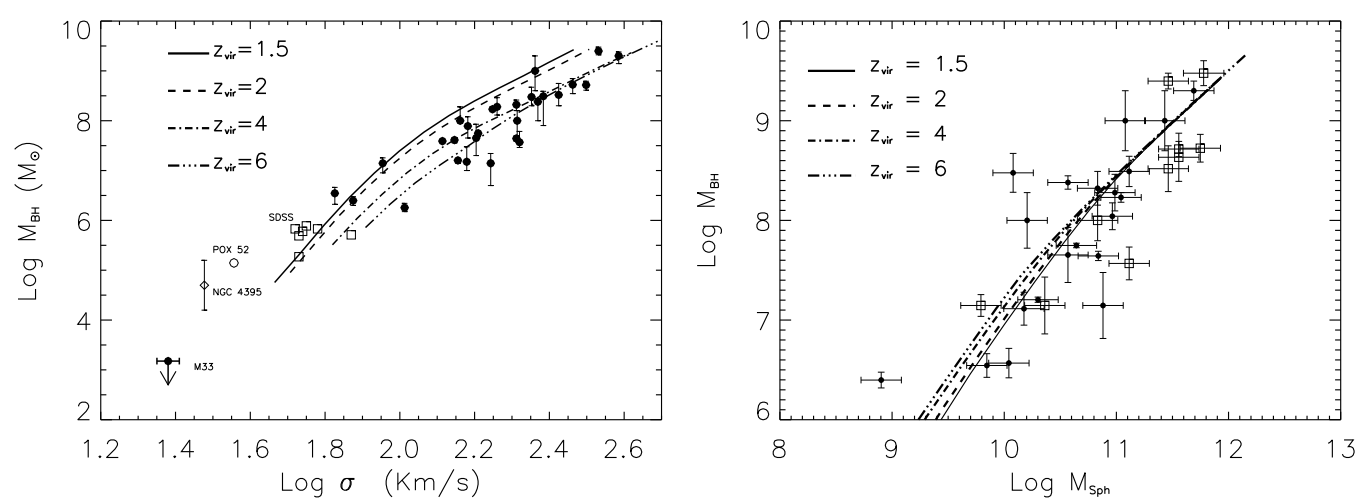

Figure 5: Left: The $M_{\mathrm{BH}}-\sigma$ relation from our model for different virialization redshifts compared with observational data. Filled circles represents nearby galaxies with dynamical measurements of the BH mass (Tremaine et al. 2002) as well as the upper limit for M33 (Gebhardt et al. 2001). Open symbols refer to galaxies with $\mathrm{BH}$ masses estimated from the $\mathrm{H} \beta$ linewidth-luminosity-mass relation (see Cirasuolo et al. 2004). Right: Predicted $M_{\mathrm{BH}}-M_{\text {star }}$ relation for different virialization redshifts compared with observational data from Häring \& Rix (2004).

We have also pointed out an impressively tight correspondence between the virial velocities, $V_{\text {vir }}$, controlled by the dynamics of dark halos, and the stellar velocity dispersions, $\sigma$, that feel the effect of dissipative baryon loading. A straightforward comparison of the virial velocity distribution, implied by the standard hierarchical clustering scenario, with the observed velocity dispersion function of spheroidal galaxies in the SDSS survey, shows that the two functions match remarkably well for a constant ratio $\sigma / V_{\text {vir }} \simeq 0.55$. This strongly suggests that, at least in the dense central regions of galaxies, on one side the star-formation occurred on a timescale shorter than the freefall time and, on the other side, the stellar dynamics has not been substantially perturbed by later merging events.

Adopting the above ratio $\sigma / V_{\text {vir }}$, the $\mathrm{ABC}$ model perfectly reproduces the observed relationships between dynamical and photometric properties which define the Fundamental Plane of elliptical galaxies.

Finally, the model accounts for the local SMBH mass function for $M_{\mathrm{BH}}>10^{7} M_{\odot}$, and for the the $M_{\mathrm{BH}}-\sigma$ relation. It predicts a steepening at $\sigma \leq 150 \mathrm{~km} / \mathrm{s}$ as the $\mathrm{SMBH}$ growth is hindered by the combined effect of SNe heating and decreased radiation drag. It also predicts the $M_{\mathrm{BH}}-M_{\mathrm{star}}$ relation to be redshift independent and its intrinsic scatter to be minimum, in agreement with recent observations.

\section{References}

[1] Alexander D.M. et al., 2003, AJ, 125, 383

[2] Baugh, C.M. et al., 2003, in: The Masses of Galaxies at Low and High Redshift R. Bender, A. Renzini, (eds. Berlin: Springer)

[3] Bernardi, M., et al., 2003, AJ, 125, 1849

[4] Cirasuolo M., Shankar F, Granato G.L., De Zotti G., Danese L., 2004, submitted to ApJ 
[5] Cole S., et al., 2001, MNRAS, 326, 255

[6] Daddi, E., et al., 2003, ApJ, 588, 50

[7] Dekel A. \& Silk J., 1986, ApJ, 303, 39

[8] D’Odorico V., Cristiani S., Romano D., Granato G.L., Danese L., 2004, MNRAS, 351, 976

[9] Fabian A.C. 1999, MNRAS, 308, L39

[10] Fan X., Strauss M.A., Richards G.T., et al. 2001, AJ, 121, 31

[11] Ferrarese L. \& Merritt D., 2000, ApJ, 539, L9

[12] Ferrarese L., 2002, ApJ, 578, 90

[13] Fontana, A., et al., 2004, A\&A, in press, [astro-ph/0405055]

[14] Gebhardt K., et al. 2000, ApJ, 539, L13

[15] Gebhardt, K., et al., 2001, AJ, 122, 2469

[16] Gerhard, O., Kronawitter, A., Saglia, R.P. \& Bender, R., 2001, AJ, 121, 1936

[17] Granato G.L., De Zotti G., Silva L., Bressan A., Danese L., 2004, ApJ, 600, 580

[18] Haehnelt M.G., Natarajan P., Rees M.J., 1998, MNRAS, 300, 817

[19] Häring, N. \& Rix, H.-W., 2004, ApJ, 604, 89

[20] Kashikawa, N., et al. 2003, AJ, 125, 53

[21] Kauffmann G., et al., 1998, MNRAS, 303, 188

[22] Kawakatu N. \& Umemura M. 2002, MNRAS, 329, 572

[23] Marconi A. \& Hunt L. 2003, ApJL, 589, L21

[24] McLure R.J. \& Dunlop J.S., 2002, NMRAS, 331,795

[25] Press, W.H., \& Schechter, P. 1974, ApJ, 187, 425

[26] Scott S.E., Fox, M.J., Dunlop, J.S., et al. 2002, MNRAS, 331, 817

[27] Shankar, F., Salucci, P., Granato, G.L., De Zotti, G. \& Danese L., 2004, MNRAS, 354, 1020

[28] Sheth, R.K. \& Tormen, G., 2002, MNRAS, 329, 61

[29] Sheth, R.K. et al., 2003, ApJ, 594, 225

[30] Silk J. \& Rees M.J., 1998, A\&A, 331, L1

[31] Silva L., Granato G.L., Bressan A., Danese, L. 1998, ApJ, 509, 103

[32] Silva L., De Zotti G., Granato G.L., Maiolino R., Danese, L., 2004, MNRAS in press, [astro-ph/0412340]

[33] Tecza M., Baker A.J., Davies R.I., Genzel R., Lehnert M.D., Eisenhauer F., et al., 2004, ApJ, 605, 109

[34] Zhao, D.H., Mo, H.J., Jing, Y.P. \& Börner G., 2003, MNRAS, 339, 12 